\title{
Robotic-assisted laparoscopic sacrocolpopexy: Initial Canadian experience
}

Yunwei Zhao; Blair St. Martin

Division of Urology, Department of Surgery, University of Alberta, Edmonton, AB, Canada

Cite as: Can Urol Assoc J 2020 January 20; Epub ahead of print. http://dx.doi.org/10.5489/cuaj.6121

Published online January 20, 2020

$* * *$

\section{Abstract}

Introduction: Abdominal sacrocolpopexy provides effective, long-term outcomes for apical pelvic organ prolapse. The introduction of robotic-assisted laparoscopic sacrocolpopexy (RALS) has mitigated the risks of abdominal surgery. This study aims to evaluate the preoperative patient characteristics, intraoperative surgical parameters, and postoperative outcomes of RALS, which has not been previously performed in Canada.

Methods: A retrospective chart review of 47 patients who have undergone RALS from 20162018 by a single surgeon at a tertiary care hospital in Canada was completed.

Results: This case series had a mean age of 60.2 years, body mass index of $28.3 \mathrm{~kg} / \mathrm{m}^{2}$, and Charlson Comorbidity Index of 2.0. Preoperatively, 13 (28\%), 23 (49\%), and 11 (23\%) patients had Baden-Walker grade 2, 3, and 4 apical prolapse, respectively. Intraoperatively, 45 (96\%) underwent concomitant procedures, including 36 (77\%) with adnexal surgery, 32 (68\%) with anti-incontinence surgery, and $25(53 \%)$ with hysterectomy. Intraoperative complications included one ureteric injury, two bladder injuries, and three vaginotomies. The mean robotic console time, surgery time, and total operating room times were 125.6, 148.6, and 190.6 minutes, respectively. Postoperatively, data was analyzed for $32(68 \%)$ patients with greater than 12 weeks followup. There was no recurrence of apical prolapse on exam after a mean followup of 60.1 weeks. Seven $(22 \%)$ patients experienced symptomatic prolapse in the posterior compartment. There were two grade 3 Clavien Dindo complications, including osteomyelitis and mesh exposure.

Conclusions: RALS can be safely and effectively performed with other pelvic procedures with good outcomes. Concurrent posterior repairs may be considered in select patients. 


\section{Introduction}

Pelvic organ prolapse is a common condition and the prevalence is $40 \%$ to $60 \%$ in women older than 50 years of age. ${ }^{1}$ The variation in prevalence depends on the method of reporting and can be as low as $2.9 \%$ when reported subjectively as opposed to $41 \%$ based on objective physical exams. ${ }^{2,3}$ Nonetheless, the overall lifetime risk of prolapse surgery is estimated to be $12.6 \%{ }^{4}$ Often, patients do not report symptoms until the prolapse extends beyond the hymenal ring, classified as an advanced stage of prolapse. ${ }^{5}$ The abdominal sacrocolpopexy (ASC) has been considered the "gold standard" for advanced apical prolapse repair. ${ }^{1}$ Compared to native tissue vaginal repair, ASC has been demonstrated in multiple randomized control trials to be superior in efficacy. ${ }^{6-8}$

The technique of ASC was first introduced by Lane in 1962 and modified by subsequent generations. ${ }^{9}$ Unfortunately, open abdominal surgeries present high morbidity to the patient, including a larger abdominal incision, increased blood loss, and longer convalescence. The advent of minimally invasive surgery in recent decades has mitigated these issues.

Laparoscopic sacrocolpopexy was introduced in $1994 .{ }^{10}$ It has been shown to have comparable outcomes to ASC. ${ }^{11,12}$ However, the limited degrees of freedom, two-dimensional vision, and steep learning curve associated with laparoscopic suturing have limited the widespread adoption of this approach. ${ }^{13}$ With the arrival of the da Vinci Surgical System (Intuitive Surgical Inc., CA, USA), robotic-assisted laparoscopic sacrocolpopexy (RALS) has been performed since 2004 and has improved on the shortcomings of conventional laparoscopic surgery. ${ }^{14}$

Robotic assistance provides increased magnification, three-dimensional vision, physiologic tremor filtering, and seven degrees of freedom to enhance surgeon ergonomics and simplify complex tasks of vaginal dissection and laparoscopic suturing. ${ }^{15}$ RALS compares favorably to ASC and is associated with decreased blood loss, shorter hospital stay, and decreased costs. ${ }^{16-18}$ These advantages have quickly enabled RALS to become the new standard of care in robotic centers around the world.

To our knowledge, RALS has not been previously performed in Canada. This study aims to evaluate the preoperative patient characteristics, intraoperative surgical parameters, and postoperative outcomes of this surgery. We reviewed our initial clinical experience and compared it to contemporary literature to demonstrate its safety and efficacy at a Canadian tertiary care center.

\section{Methods}

This study has been approved by the Health Research Ethics Board at the University of Alberta in Edmonton, Alberta. A retrospective chart review of RALS was completed using prospectively collected data. A total of 47 patients who underwent RALS from August 2016 to December 2018 were identified using the billing code for sacrocolpopexy, with the last patient follow-up in April 
2019. All surgeries were consecutive RALS performed by a single surgeon at a tertiary care center in Northern Alberta. Most patients had concomitant pelvic and anti-incontinence surgeries performed prior to and after sacrocolpopexy, respectively. Anti-incontinence procedure was added if the patient had symptomatic stress urinary incontinence confirmed either at the time of cystoscopy with cough stress test or on urodynamics.

Detailed preoperative patient information was extracted from the patients' electronic medical records (HealthQuest ${ }^{\mathrm{TM}}$ ) at the time of their preoperative visit, which included demographic information, medical history, surgical history, and examination using the Baden Walker scale. Demographic information included age, body mass index (BMI), parity, postmenopausal status, smoking history, and the use of systemic or local hormone therapy. Medical history was used to derive the Charlson Comorbidity Index according to the published algorithm. ${ }^{19}$ Surgical history included prior abdominal surgery, hysterectomy, prolapse or antiincontinence surgery. The American Society of Anesthesiologist score was obtained from the anesthesia records on the day of operation.

Our primary outcome was the rate of recurrent apical prolapse assessed by objective examination. Numerous secondary outcomes were measured. Secondary intraoperative outcomes were obtained from the surgeon's operative notes and included type of concomitant procedure, total operating room time, surgical time, robotic console time, estimated blood loss, and intraoperative complication rate. Secondary postoperative outcomes were obtained from hospital records as well as patients' electronic medical records during follow-up visits and included hemoglobin concentration change, length of hospital stay, 90-day complication rate, patient satisfaction questionnaire score, re-intervention rate, and long-term complication rate.

Patients were followed at 6 weeks postoperatively, when complications arose, and at a final visit arranged by the surgeon, typically at greater than 3 months. At each follow-up visit, the severity of prolapse was assessed objectively using either the Baden Walker scale or the Pelvic Organ Prolapse Quantification (POPQ) system by one of the authors. Subjective outcomes were assessed by the Patient Global Impression of Improvement (PGI-I) scale, as well as asking the question of "Would you recommend this surgery to another friend". ${ }^{20}$

Descriptive statistics were used in this study, where numerical values were presented using the mean and standard deviation. Student's t-test and ANOVA were used to determine the significance between two and three means, respectively. Z-test was used to determine the significance between proportions.

\section{Technique}

Although specific techniques vary, the general principles of RALS have been reviewed previously. ${ }^{21}$ The robotic port placement is a five port configuration placed in dorsal lithotomy position, including a $12 \mathrm{~mm}$ periumbilical disposable port placed $20 \mathrm{~cm}$ above the symphysis 
pubis, two $8 \mathrm{~mm}$ robotic ports placed on the left, one additional robotic port and a $12 \mathrm{~mm}$ assistant port placed on the right (Fig. 1). All ports were placed at least $8 \mathrm{~cm}$ apart to avoid clashing of instruments. After parallel docking of the da Vinci Surgical System (Intuitive Surgical, CA, USA), robotic monopolar scissors, bipolar forceps, and ProGrasp ${ }^{\mathrm{TM}}$ forceps were attached to robotic arms 1,2 , and 3 , respectively.

The peritoneum overlying the sacral promontory was incised and the anterior longitudinal ligament of the sacrum was exposed (Fig. 2A). The peritoneal incision was extended inferiorly along the lateral border of the rectum. A malleable retractor ( $1.5 \times 13$ inches, Canadian Hospital Specialties, ON, Canada) was used for vault manipulation. The vesicovaginal space was developed anteriorly to the level of the bladder trigone and the rectovaginal space was developed to just above the levator ani (Fig. 2B). The Restorelle Y mesh (Coloplast, MN, USA) was fashioned according to the anatomical dissection and secured to the vaginal vault using 2-O PDS or Prolene sutures. Three to five sutures were used on each side of the mesh with interrupted sutures placed at the distal and proximal end of the mesh and running sutures on the side of the mesh. PDS sutures were preferentially used near the bladder trigone and the vaginal vault if concomitant hysterectomy was performed to decrease the risk of mesh erosion. The sacral arm of the mesh was secured to the anterior longitudinal ligament using two O Prolene sutures (Fig. 2C). Finally, peritoneal closure was accomplished with a barbed absorbable suture to prevent the risk of bowel herniation (Fig. 2D).

\section{Results}

Preoperative patient information is presented in Table 1. The average age was 60.2 years with a mean BMI of $28.3 \mathrm{~kg} / \mathrm{m}^{2}$. Mean parity was 2.7 and mean Charlson Comorbidity Index was 2.0. Of the 47 women, 40/47 (85\%) were postmenopausal and 34/47 (72\%) have never smoked. While most women were not taking systemic hormone therapy preoperatively, 30/47 (64\%) were on vaginal estrogen. In terms of surgical history, 35/47 (74\%) had previous abdominal surgery, 21/47 (45\%) had previous hysterectomy, 13/47 (28\%) had previous prolapse surgery, including $6 / 47(13 \%)$ with apical repair, and 10/47 (21\%) had previous anti-incontinence surgery. At the preoperative assessment, 34/47 (72\%) were classified as grade 3 or 4 prolapse, while the remaining 13/47 (28\%) had grade 2 prolapse.

Intraoperative surgical parameters are presented in Table 2. Of the 47 women, 42 (89\%) had RALS with suspension of both the anterior and posterior vagina, 4 (9\%) had suspension of only the anterior vagina, done in patients without a significant posterior compartment prolapse, and $1(2 \%)$ had sacrohysteropexy. Of the concomitant pelvic surgeries, $36(77 \%)$ had adnexal surgery including salpingectomy/oophorectomy, 32 (68\%) had anti-incontinence surgery, including 29 (62\%) with Burch colposuspension, and 25 (53\%) had hysterectomy. There was no estimated blood loss greater than $100 \mathrm{~mL}$ and no conversion to laparotomy. Intraoperative 
complications included 3 vaginotomies (6\%), 2 bladder injuries (4\%), and 1 ureteric injury (2\%). The mean total operating room time from patient entry to exit was $190.6 \mathrm{~min}$; surgery time from skin incision to closure was $148.6 \mathrm{~min}$; and robotic console time was $125.6 \mathrm{~min}$.

Postoperative outcomes are presented in Table 3, which analyzed $32(68 \%)$ patients with a follow-up duration of greater than 12 weeks. The mean duration of follow-up was 60.1 weeks $(19-130)$. In the recovery period, the mean decrease in hemoglobin was $23.1 \mathrm{~g} / \mathrm{L}$ with a mean decrease in hematocrit of $6.9 \%$, and themean duration of hospital stay was 1.4 days. On objective exam, there was no evidence of apical prolapse greater than grade 1 on the Baden Walker scale. The mean point $\mathrm{C}$ distance was $-7.3 \mathrm{~cm}$ when assessed by the POPQ system. Of the 4 patients with suspension of only the anterior vagina, 2 had follow-up greater than 12 weeks and they had no recurrence of prolapse in any compartment. Patient satisfaction was assessed in 26/32 (81\%) of patients. On the PGI-I questionnaire, 22/26 (85\%) reported a score of 1 (very much better) or 2 (much better), and 24/26 (92\%) would recommend this surgery to another friend. The reintervention rate for prolapse of any compartment was $7 / 32(22 \%)$, due to recurrences of grade 2 prolapse in the anterior and posterior compartment (2) and in the posterior compartment alone (5). Interventions included surgical referral to urogynecology (3), pelvic floor physiotherapy (2), pessary fitting (1), and other investigations (1). Three of $29(10 \%)$ required insertion of tension free vaginal tape despite having had concurrent Burch colposuspension with RALS after a mean duration of 12 months.

Short and long-term postoperative complications are presented in Table 4. Complications within 90 days were graded using the Clavien-Dindo system. Grade 1 complications included pain (9), prolonged catheterization (6), stress urinary incontinence (3 de novo, 2 recurrent), gastrointestinal symptoms (2), rectal prolapse (1), overactive bladder (1), and seroma (1). Grade 2 complications included urinary tract infection (3), overactive bladder (2), blood transfusion (1), and infected hematoma (1). There were two grade 3 complications, including one mesh exposure requiring excision by urogynecology and one sacral osteomyelitis requiring drainage of abscess by interventional radiology. Complications beyond 90 days included suture exposure (2), pelvic pain (1), voiding dysfunction (1), and defecatory dysfunction (1). These suture exposures were asymptomatic and excised during examination, while the rest were managed conservatively. Table 5 compares the results between the first and last 10 cases as well as between the different grades of apical prolapse. Comparing earlier versus later cases, there were no significant differences in terms of intraoperative complications, total operating room time, change in hemoglobin, hospital stay, 90-day complications, as well as subjective and objective outcomes. The only significant difference was the duration of follow-up ( 87.8 vs. 33.4 weeks). Comparing between the different grades of apical prolapse, there were no differences in any of the parameters assessed. 


\section{Discussion}

Our findings demonstrated that RALS can be safely and efficiently performed at a tertiary care center in Canada with good outcomes at a mean follow-up of 60.1 weeks. These findings were consistent with what was previously published in the literature.

In 2014, Serati published the largest systematic review on RALS, consisting of 27 studies from 2006 to 2013 with a total of 1488 patients. ${ }^{22}$ They found a median operative time of 194 min $(75-537)$, estimated blood loss of $50 \mathrm{~mL}(10-1000)$, and hospital stay of 2 days $(0-50)$, which were similar to our findings. Operative times varied across different studies and reflected the surgical volume and execution of concomitant procedures, including the performance of hysterectomy and anti-incontinence surgery in 38\% and 33\% of all cases, respectively. Given that our proportion of concomitant surgeries were higher than these figures, it is likely that our procedures were performed more efficiently than most of the studies in their review. This may be attributed to the use of a dedicated robotic surgical team at our center, which has been previously shown to reduce operative time by as much as 26 min for RALS. ${ }^{23}$

In terms of objective cure of apical prolapse, Serati et al. reported a range between $97 \%$ and $100 \%$. When considering the four studies with a medium-term follow-up of more than two years, the cure rate was $99.2 \% .^{22}$ In a separate systematic review by Hudson et al. with 13 studies and 577 patients, they found a cure rate of $98.6 \%$ at a mean follow-up of 26.9 months. ${ }^{24}$ Similarly, a recent large RALS series with 196 patients found an apical recurrence rate of $2 \%$ after a mean follow-up of 9 months. ${ }^{25}$ These results are similar to our finding of $100 \%$ apical cure rate at a mean follow-up of 60.1 weeks. Postoperative outcomes were limited to patients with follow-up of greater than 12 weeks to more accurately assess recurrence and adverse events. Compared to objective outcomes, only a handful of studies reported subjective outcomes and satisfaction. Numerous validated questionnaires were used but the reporting of subjective outcomes were heterogenous. ${ }^{26-29}$ Overall, there was an improvement of postoperative questionnaire scores compared to preoperative scores. ${ }^{27,28}$ In our study, we chose to use the PGI-I as well as the question of recommendation to friends during follow-up due to their ease of administration and found reasonably good patient satisfaction (Table 3 ). These findings were similar to what was reported in the Hudson review where seven studies reported postoperative patient satisfaction of greater than $90 \% .^{24}$

Both previous systematic reviews found a reoperation rate of $3.3 \%$ for recurrent prolapse, mainly in non-apical compartments. ${ }^{22,24}$ Specifically, most of the surgical reoperations were in the posterior compartment with $2.1 \%$ being posterior colporrhaphies in the Serati review. ${ }^{22}$ These findings were confirmed by a recent large RALS series and highlighted the possibility of a less aggressive dissection in the rectovaginal space. ${ }^{25}$ In our study, we noted seven patients with recurrent prolapse symptoms in the posterior compartment that required additional intervention. Our technique involved dissection of the rectovaginal space by 6 to $8 \mathrm{~cm}$ without reaching the 
perineal body to decrease the risk of rectal injury and postoperative anorectal symptoms that can result from an aggressive dissection. Hence, distal rectoceles are not addressed by this technique and some of these patients may require additional vaginal reconstructive surgery. Of note, a recent study correlated the reduction of composite anatomic failure, specifically in the posterior compartment, with surgical reduction to a normal $(<4 \mathrm{~cm})$ genital hiatus $(\mathrm{GH})$ in women with a wide $\left(>4 \mathrm{~cm}\right.$ ) preoperative $\mathrm{GH} .{ }^{30}$ Thus, it may be prudent to consider concomitant perineorrhaphy at the time of RALS in women with a persistently wide GH.

In terms of intraoperative complications, we noted a slightly higher proportion of vaginotomies, bladder and ureteric injuries compared to the Serati review, published as $1 \%, 2 \%$, $<1 \%$, respectively. ${ }^{22} \mathrm{We}$ noted no bowel injury or conversion to laparotomy. This may reflect the initial learning curve of the procedure as the first cases performed in Canada as well as the small total number of patients in this series. However, our results compared favorably to the study by Anand et al. which noted a vaginotomy, cystotomy, bowel injury, and conversion rate of $24 \%$, $10 \%, 4 \%$, and $12 \%$ respectively. ${ }^{31}$ Nonetheless, our complications were recognized and repaired immediately with no long-term sequelae.

In terms of postoperative complications, most were Clavien-Dindo grade 1 or 2 and resolved with conservative or medical therapy (Table 4). The most common postoperative complication was pain, which resulted in additional investigations or visits. These patients were reassured, and symptoms resolved with regularly scheduled analgesia. We attribute our decrease in hemoglobin to hemodiluation as reflected in the decrease in hematocrit and venous oozing from our dissection sites after the pneumoperitoneum was removed. There was one rare complication of osteomyelitis noted in our study in a patient with preexisting neurogenic bladder who did not continue with clean intermittent catheterization postoperatively. This resulted in urinary retention, acute kidney injury, multidrug resistant bacteremia, sacral osteomyelitis on MRI at 3 months, and drainage of abscess at 8 months postoperatively without the need for mesh removal.

There was one case of mesh exposure that required surgical excision at 3 months postoperatively, which was similar to the $2 \%$ mesh complication rate published in the Serati review. ${ }^{22}$ Of note, many of our patients had concomitant hysterectomy, a known risk factor for mesh exposure. ${ }^{32} \mathrm{We}$ attribute our low mesh complication rate to our surgical technique of mesh attachment away from the vault line, selective use of delayed absorbable rather than permanent sutures, and use of an ultra-light weight type I polypropylene mesh. The extended CARE trial had shown that mesh complications increase over time and can reach $10.5 \%$ at 7 years after an ASC..$^{33}$ On the other hand, a long term RALS series with a median follow-up of 6 years demonstrated a much lower surgical intervention rate of $2.7 \%$ for mesh complications and provided assurance of long-term safety for this minimally invasive technique. ${ }^{34}$ 
Limitations of this study included the retrospective nature of the study design. As such, there was no standardized preoperative objective/subjective assessment and postoperative follow-up protocol, which limited the determination of surgical improvement. Another limitation was the shorter mean duration of follow-up. We would expect that the apical failure and mesh complication rate to increase over time. Given the nature of our tertiary referral center, we would also expect that some patients were followed by local practitioners instead and these data were not captured. Strengths of this study included the performance of RALS by a single surgeon with the same surgical technique and an experienced robotic team. Outcomes measured included objective and subjective assessments in addition to re-intervention rate. The pragmatic nature of the study was reflected in the variety of procedures performed concomitantly with RALS.

\section{Conclusions}

RALS can be safely and efficiently performed with other pelvic procedures. There is good apical support, high patient satisfaction, and low rate of complication after a mean follow-up of 60.1 weeks. The posterior compartment is more likely to have symptomatic recurrence and concurrent posterior repair may be considered in select patients.

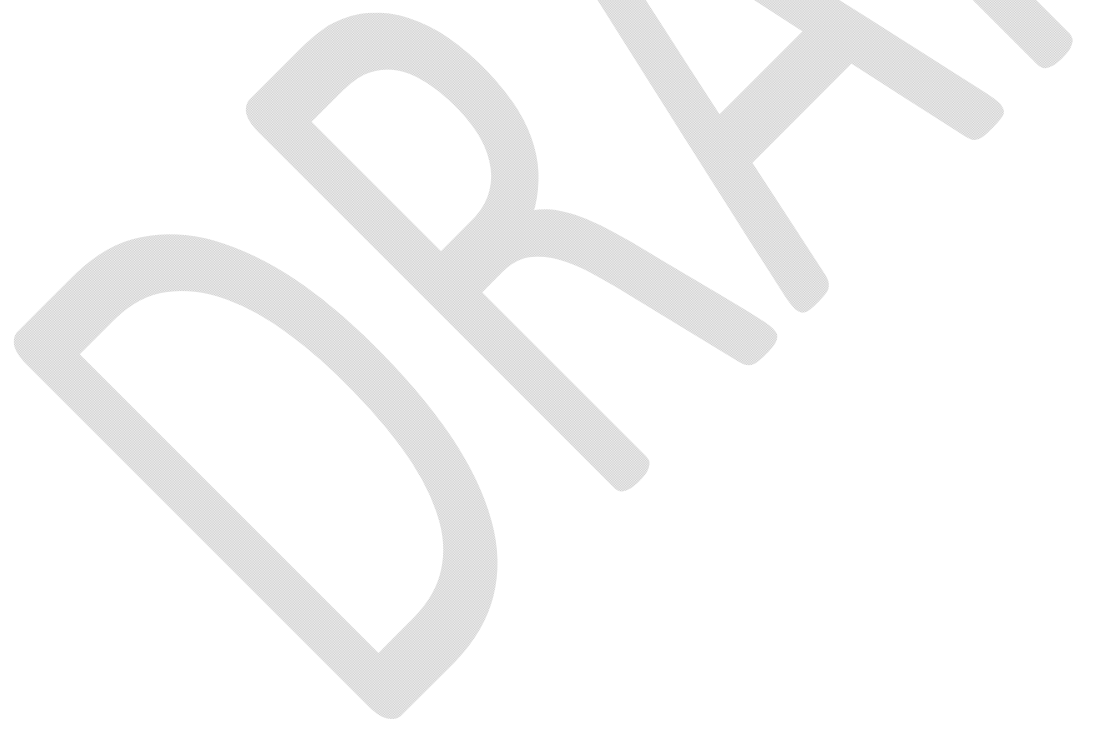




\section{References}

1. Maher C, Feiner B, Baessler K, et al. Surgery for women with apical vaginal prolapse. Cochrane Database Syst Rev 2016;(10):CD012376.

2. Nygaard I, Barber MD, Burgio KL, et al. Prevalence of symptomatic pelvic floor disorders in US women. JAMA 2008;300:1311-6.

3. Hendrix SL, Clark A, Nygaard I, et al. Pelvic organ prolapse in the Women's Health Initiative: Gravity and gravidity. Am J Obstet Gynecol 2002;186:1160-6.

4. $\mathrm{Wu} \mathrm{JM}$, Matthews CA, Conover MM, et al. Lifetime risk of stress urinary incontinence or pelvic organ prolapse surgery. Obstet Gynecol 2014;123:1201-6.

5. Auwad W, Freeman RM, Swift S. Is the pelvic organ prolapse quantification system (POPQ) being used? A survey of members of the International Continence Society (ICS) and the American Urogynecologic Society (AUGS). Int Urogynecol J Pelvic Floor Dysfunct 2004;15:324-7.

6. Benson JT, Lucente V, McClellan E. Vaginal versus abdominal reconstructive surgery for the treatment of pelvic support defects: A prospective randomized study with longterm outcome evaluation. Am J Obstet Gynecol 1996;175:1418-22.

7. Lo TS, Wang AC. Abdominal colposacropexy and sacrospinous ligament suspension for severe uterovaginal prolapse: A comparison. J Gynecol Surg 1998;14:59-64.

8. Maher CF, Qatawneh AM, Dwyer PL, et al. Abdominal sacral colpopexy or vaginal sacrospinous colpopexy for vaginal vault prolapse: A prospective randomized study. $\mathrm{Am}$ J Obstet Gynecol 2004;190:20-6.

9. Lane FE. Repair of posthysterectomy vaginal-vault prolapse. Obstet Gynecol 1962;20:727.

10. Nezhat $\mathrm{CH}$, Nezhat F, Nezhat C. Laparoscopic sacral colpopexy for vaginal vault prolapse. Obstet Gynecol 1994;84:88-8.

11. Paraiso MF, Walters MD, Rackley RR, et al. Laparoscopic and abdominal sacral colpopexies: A comparative cohort study. Am J Obstet Gynecol 2005;192:1752-8.

12. Freeman RM, Pantazis K, Thomson A, et al. A randomised controlled trial of abdominal versus laparoscopic sacrocolpopexy for the treatment of posthysterectomy vaginal vault prolapse: LAS study. Int Urogynecol J 2013;24:377-84.

13. Mustafa S, Amit A, Filmar S, et al. Implementation of laparoscopic sacrocolpopexy: Establishment of a learning curve and short-term outcomes. Arch Gynecol Obstet 2012;286:983-8.

14. Elliott DS, Frank I, Dimarco DS, et al. Gynecologic use of robotically assisted laparoscopy: Sacrocolpopexy for the treatment of high-grade vaginal vault prolapse. Am J Surg 2004;188:S52-6.

15. Lee RK, Mottrie A, Payne CK, et al. A review of the current status of laparoscopic and robot-assisted sacrocolpopexy for pelvic organ prolapse. Eur Urol 2014;65:1128-37.

16. Elliott CS, Hsieh MH, Sokol ER, et al. Robot-assisted versus open sacrocolpopexy: A cost-minimization analysis. J Urol 2012;187:638-43. 
17. Siddiqui NY, Geller EJ, Visco AG. Symptomatic and anatomic 1-year outcomes after robotic and abdominal sacrocolpopexy. Am J Obstet Gynecol 2012;206:435.e1-5.

18. Geller EJ, Siddiqui NY, Wu JM, et al. Short-term outcomes of robotic sacrocolpopexy compared with abdominal sacrocolpopexy. Obstet Gynecol 2008;112:1201-6.

19. Quan H, Li B, Couris CM, et al. Updating and validating the Charlson comorbidity index and score for risk adjustment in hospital discharge abstracts using data from 6 countries. Am J Epidemiol 2011;173:676-82.

20. Srikrishna S, Robinson D, Cardozo L. Validation of the Patient Global Impression of Improvement (PGI-I) for urogenital prolapse. Int Urogynecol J 2010;21:523-8.

21. Clifton MM, Pizarro-Berdichevsky J, Goldman HB. Robotic female pelvic floor reconstruction: A review. Urology 2016;91:33-40.

22. Serati M, Bogani G, Sorice P, et al. Robot-assisted sacrocolpopexy for pelvic organ prolapse: A systematic review and meta-analysis of comparative studies. Eur Urol 2014;66:303-18.

23. Carter-Brooks CM, Du AL, Bonidie MJ, et al. The impact of a dedicated robotic team on robotic-assisted sacrocolpopexy outcomes. Female Pelvic Med Reconstr Surg 2018;24:13-6.

24. Hudson CO, Northington GM, Lyles RH, et al. Outcomes of robotic sacrocolpopexy: A systematic review and meta-analysis. Female Pelvic Med Reconstr Surg 2014;20:252-60.

25. Gupta P, Ehlert M, Bartley J, et al. Perioperative outcomes, complications, and efficacy of robotic-assisted prolapse repair: A single institution study of 196 patients. Female Pelvic Med Reconstr Surg 2018;24:408-11.

26. Geller EJ, Parnell BA, Dunivan GC. Robotic vs abdominal sacrocolpopexy: 44-month pelvic floor outcomes. Urology 2012;79:532-6.

27. Paraiso MF, Jelovsek JE, Frick A, et al. Laparoscopic compared with robotic sacrocolpopexy for vaginal prolapse. Obstet Gynecol 2011;118:1005-13.

28. Geller EJ, Parnell BA, Dunivan GC. Pelvic floor function before and after robotic sacrocolpopexy: one-year outcomes. J Minim Invasive Gynecol 2011;18:322-7.

29. Siddiqui NY, Geller EJ, Visco AG. Symptomatic and anatomic 1-year outcomes after robotic and abdominal sacrocolpopexy. Am J Obstet Gynecol 2012;206:435.e1-5.

30. Bradley MS, Askew AL, Vaughan MH, et al. Robotic-assisted sacrocolpopexy: Early postoperative outcomes after surgical reduction of enlarged genital hiatus. Am J Obstet Gynecol 2018;218:514.e1-8.

31. Anand M, Woelk JL, Weaver AL, et al. Perioperative complications of robotic sacrocolpopexy for post-hysterectomy vaginal vault prolapse. Int Urogynecol $J$ 2014;25(9):1193-1200.

32. Osmundsen BC, Clark A, Goldsmith C, et al. Mesh erosion in robotic sacrocolpopexy. Female Pelvic Med Reconstr Surg 2012;18:86-8.

33. Nygaard I, Brubaker L, Zyczynski H, et al. Long-term outcomes following abdominal sacrocolpopexy for pelvic organ prolapse. JAMA 2013;309:2016-24.

34. Linder BJ, Chow GK, Elliott DS. Long-term quality of life outcomes and retreatment rates after robotic sacrocolpopexy. Int J Urol 2015;22:1155-8. 


\section{Figures and Tables}

Fig. 1. Port placement for robotic-assisted laparoscopic sacrocolpopexy. Setup consists of five ports with a $12 \mathrm{~mm}$ disposable periumbilical port; two $8 \mathrm{~mm}$ reusable robotic ports on the left; one $8 \mathrm{~mm}$ reusable robotic port and one $12 \mathrm{~mm}$ disposable port on the right.

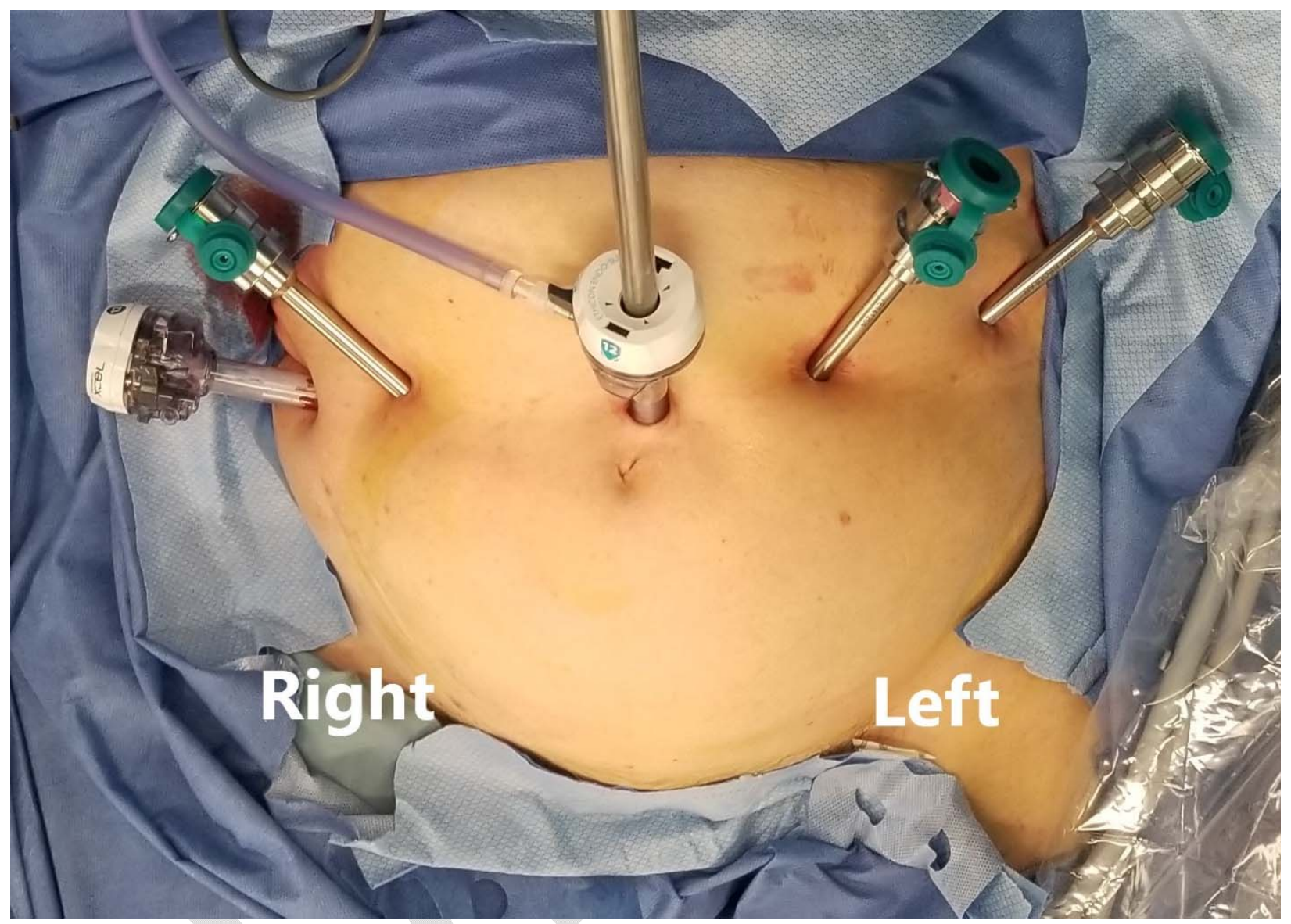


Fig. 2. Surgical technique of robotic-assisted laparoscopic sacrocolpopexy. (A) Exposure of the anterior longitudinal ligament at the sacral promontory. (B) Dissection of the anterior vesicovaginal space and posterior rectovaginal space, using a malleable vaginal manipulator. (C) Attachment of polypropylene Y mesh to the vagina and anterior longitudinal ligament. (D) Closure of peritoneum for mesh coverage.
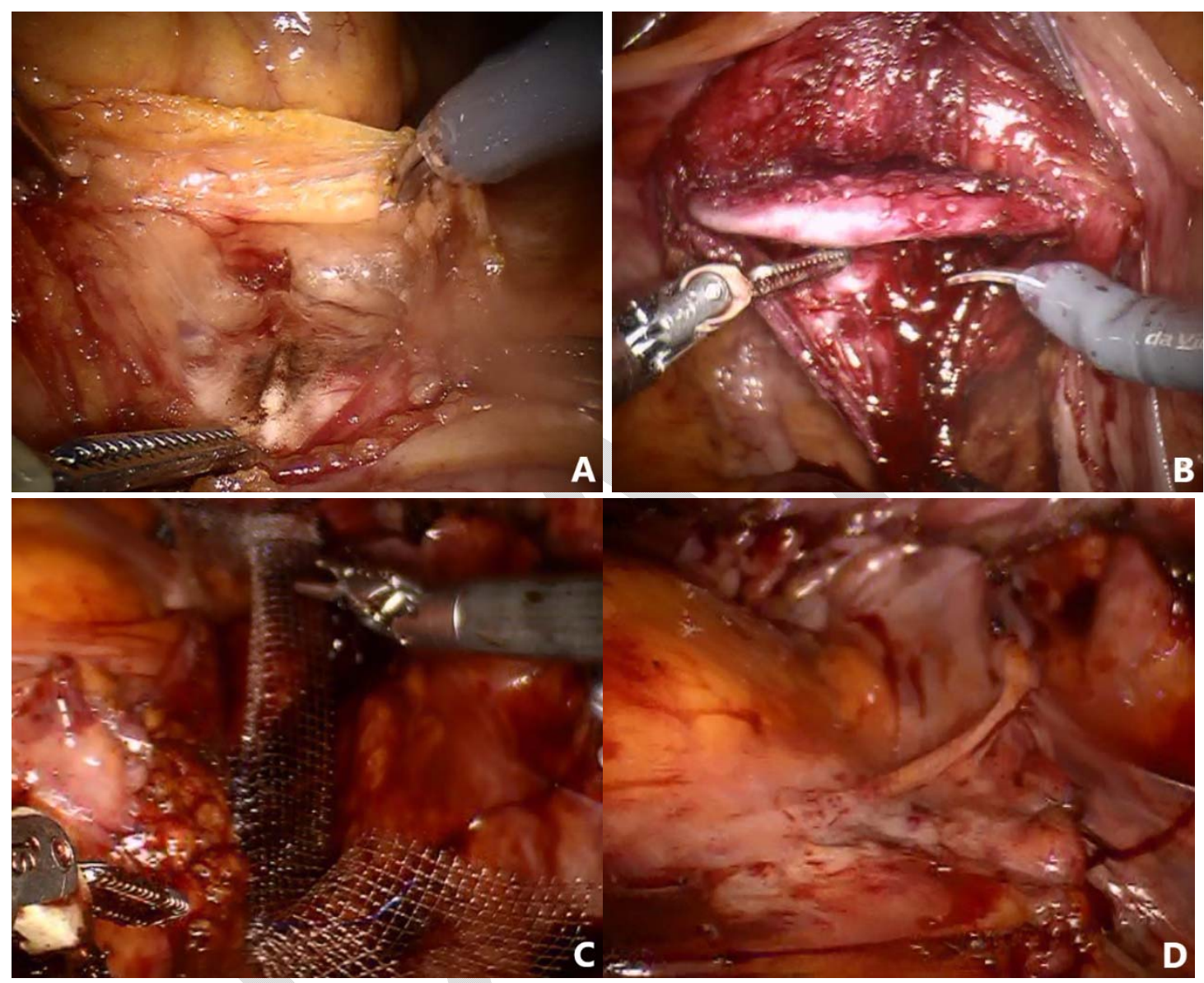


\begin{tabular}{|l|c|}
\hline Table 1. Preoperative patient information \\
\hline Characteristic* & Mean (SD) or n (\%) \\
\hline Age, year & $60.2(10.9)$ \\
\hline BMI, $\mathrm{kg} / \mathrm{m}^{2}$ & $28.3(3.7)$ \\
\hline Parity $(\mathrm{n}=35)$ & $2.7(1.1)$ \\
\hline Charlson Comorbidity Index & $2.0(1.5)$ \\
\hline ASA score & $2.0(0.6)$ \\
\hline Postmenopausal & $40(85)$ \\
\hline Preoperative hormone therapy & $3(6)$ \\
\hline Preoperative vaginal estrogen & $30(64)$ \\
\hline Smoking history: & \\
\hline Current smoker & $5(11)$ \\
\hline Previous smoker & $8(17)$ \\
\hline Never smoker & $34(72)$ \\
\hline Previous abdominal surgery & $35(74)$ \\
\hline Previous hysterectomy & $21(45)$ \\
\hline Previous prolapse surgery & \\
\hline Any prolapse surgery & $13(28)$ \\
\hline Apical prolapse surgery & $6(13)$ \\
\hline Previous anti-incontinence surgery & $10(21)$ \\
\hline Preoperative prolapse & \\
\hline Grade 2 & $13(28)$ \\
\hline Grade 3 & $23(49)$ \\
\hline Grade 4 & $11(23)$ \\
\hline
\end{tabular}

${ }^{*} \mathrm{~N}=47$ unless otherwise specified. ASA: American Society of Anesthesiologist; BMI: body mass index; SD: standard deviation. 


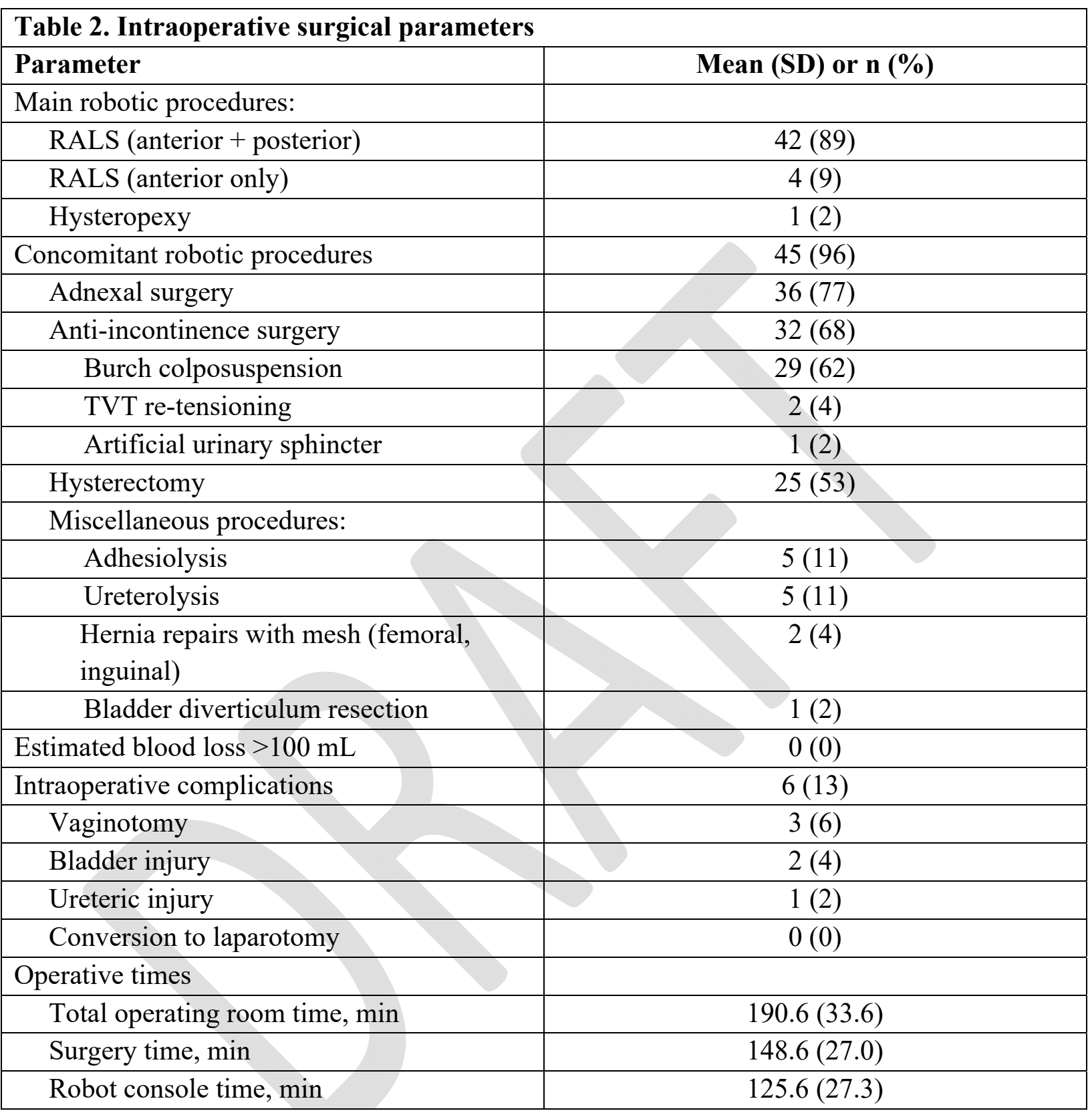

RALS: robotic-assisted laparoscopic sacrocolpopexy; SD: standard deviation; TVT: tension-free vaginal tape. 


\begin{tabular}{|l|c|}
\hline Table 3. Postoperative outcomes \\
\hline Outcome & Mean (SD) or n (\%) \\
\hline Followup & $32(68 \%)$ \\
\hline Followup $>12$ weeks $(\mathrm{n}=47)$ & $60.1(25.4)$ \\
\hline Duration of followup $(\mathrm{n}=32)$, weeks & $23.1(8.6)$ \\
\hline Recovery period & $6.9(2.7)$ \\
\hline Decrease in hemoglobin $(\mathrm{n}=40), \mathrm{g} / \mathrm{L}$ & $1.4(0.6)$ \\
\hline Decrease in hematocrit $(\mathrm{n}=40), \%$ & \\
\hline Hospital stay, days $(\mathrm{n}=47)$ & $0(0)$ \\
\hline Objective outcome & $-7.3(0.8)$ \\
\hline Apical prolapse $>$ grade $1(\mathrm{n}=32)$ & \\
\hline Point C (n=26), cm & $22(85)$ \\
\hline Patient satisfaction (n=26) & $24(92)$ \\
\hline PGI-I score 1 or 2 & $7(22)$ \\
\hline Recommend surgery to friend & $2(6)$ \\
\hline Re-intervention for any prolapse $(\mathrm{n}=32)$ & $0(0)$ \\
\hline Anterior + posterior & $5(16)$ \\
\hline Anterior only & $3(10)$ \\
\hline Posterior only & \\
\hline TVT after Burch colposuspension $(\mathrm{n}=29)$ & \\
\hline
\end{tabular}

PGI-I: Patient Global Impression of Improvement; TVT: tension-free vaginal tape. 


\begin{tabular}{|l|c|}
\hline Table 4. Postoperative complications & $\mathbf{n ~ ( \% )}$ \\
\hline Complication within 90 days (n=47) & \\
\hline Clavien Dindo grade 1 & $9(19)$ \\
\hline Pain & $6(13)$ \\
\hline Catheterization $\leq 7$ days & $5(11)$ \\
\hline Stress urinary incontinence & $2(4)$ \\
\hline Gastrointestinal symptom & $1(2)$ \\
\hline Rectal prolapse & $1(2)$ \\
\hline Overactive bladder & $1(2)$ \\
\hline Seroma & \\
\hline Clavien Dindo grade 2 & $3(6)$ \\
\hline Urinary tract infection & $2(4)$ \\
\hline Overactive bladder & $1(2)$ \\
\hline Transfusion & $1(2)$ \\
\hline Infected hematoma & \\
\hline Clavien Dindo grade 3 & $1(2)$ \\
\hline Osteomyelitis (3a) & $1(2)$ \\
\hline Mesh excision (3b) & $2(6)$ \\
\hline Complication beyond 90 days (n=32) & $1(3)$ \\
\hline Suture exposure & $1(3)$ \\
\hline Pelvic pain & $1(3)$ \\
\hline Voiding dysfunction & \\
\hline Defecatory dysfunction & \\
\hline
\end{tabular}




\begin{tabular}{|c|c|c|c|c|c|}
\hline & First 10 & Last 10 & Grade 2 & Grade 3 & Grade 4 \\
\hline $\begin{array}{l}\text { Intraoperative } \\
\text { complications }\end{array}$ & $\begin{array}{l}1 \text { ureteric } \\
\text { injury }\end{array}$ & $\begin{array}{c}1 \\
\text { vaginotomy, } \\
1 \text { cystotomy }\end{array}$ & $\begin{array}{c}1 \text { ureteric } \\
\text { injury, } 1 \\
\text { vaginotomy }\end{array}$ & $\begin{array}{c}1 \text { bladder } \\
\text { injury, } 2 \\
\text { vaginotomy }\end{array}$ & 1 cystotomy \\
\hline OR time (min) & 197.4 & 193.1 & 189 & 196.6 & 179.7 \\
\hline $\begin{array}{l}\text { Hb change } \\
(\mathrm{g} / \mathrm{L})\end{array}$ & 24.9 & 19.6 & 24.5 & 22.5 & 22.8 \\
\hline $\begin{array}{l}\text { Hospital stay } \\
\text { (days) }\end{array}$ & 1.2 & 1.3 & 1.2 & 1.4 & 1.5 \\
\hline $\begin{array}{l}\text { Clavien Dindo } \\
\geq 2\end{array}$ & $10 \%(10)$ & $20 \%(10)$ & $15 \%(13)$ & $22 \%(23)$ & $18 \%(11)$ \\
\hline $\begin{array}{l}\text { Followup } \\
\text { (weeks) }\end{array}$ & $87.8^{*}(8)$ & $33.4^{*}(5)$ & $56.6(7)$ & $63.2(18)$ & $55.6(7)$ \\
\hline PGI-I & $2.0(5)$ & $1.3(4)$ & $1.4(5)$ & $2.0(14)$ & $1.9(7)$ \\
\hline Recommend & $100 \%(5)$ & $100 \%(4)$ & $100 \%(5)$ & $86 \%(14)$ & $100 \%(7)$ \\
\hline $\mathrm{C}(\mathrm{cm})$ & $-7.4(5)$ & $-7.8(4)$ & $-7.2(5)$ & $-7.4(14)$ & $-7.3(7)$ \\
\hline $\begin{array}{l}\text { Recurrent } \\
\text { prolapse }\end{array}$ & $50 \%(8)$ & $0 \%(5)$ & $29 \%(7)$ & $22 \%(18)$ & $14 \%(7)$ \\
\hline
\end{tabular}

() denotes the number of patients with followup greater than 12 weeks in each subcategory "Statistical significance with $\mathrm{p}<0.05$. Hb: hemoglobin; OR: operating room; PGI-I: Patient Global Impression of Improvement. 